\title{
Astronomical magnitudes in the Santa María la Asunción codex
}

\author{
Alberto Camacho ${ }^{1}$ \\ ${ }^{I}$ Department of Basics Sciences of the Technological Institute of Chihuahua II-TecNM, México
}

\begin{abstract}
In this document are the results of the research to the codex Santa María la Asunción y Vergara, it contains the plots of land that are located in the acolhua region of Texcoco, Mexico. The plots show their perimeter size in $\mathrm{T}$ units. It is important that the size of length and area correspond both to the astronomical dimensions of the planet movements that astronomical acolhuas people observed regularly. The planetary revolutions establish correlations between them, which determine a control of their own movements. It also presents the twentieth-thirteenth numeric system, which supports the activities that allowed the measurement of lots, astronomical observations and building monuments. This research was developed from a multidisciplinary vision highlighting astronomy, mathematics (arithmetic and elementary geometry) and surveying.
\end{abstract}

Keywords - Mesoamerican cultures, Santa María la Asunción codex, surveyor rules

\subsection{Approaches to the codex}

\section{BACKGROUND}

About 386 plots were drawn in Santa María Asunción y Vergara codex by surveyors of acolhua culture of Texcoco region, located in the northeast of Mexico City. The codex was elaborated around 1534-1544 A.D with the purpose of having a land registry. The researchers group with the command of María del Carmen Jorge [1] (1) calculated the $T^{2}$ area of the entirety of plots contained there, mainly 122 regular lands with right angles in their vertex, and 261 irregular lands, quadrilateral, with no dimension or scale and without showing the angles, in this point having geometrical difficulty to measure them. Three of them are shown in the images of Figure 1. In the support information there are 88 square plots of $20 \times 20 \mathrm{~T}$, as well as 10 rectangular plots of 10x20 T and other of different dimensions. At the end of the report it is shown the measure of an area called El Topote, located in the same acolhua zone of the Topotitla location. The plot's area, approximately 1.24 hectares, was measured from an aerial photograph of the place taken around 1965.

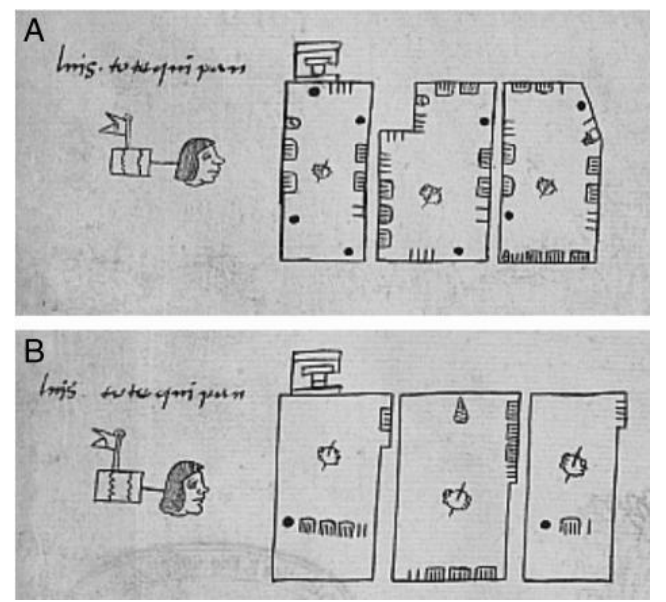

Figure 1. Portion of Santa María la Asunción y Vergara codex that shows irregular lots with numerical information of the perimeter, the ones from upside, and supposedly from the area, the down side.

Source: http://www.ncbi.nlm.nih.gov/pmc/articles/PMC3174618/

Every area was calculated and verified from a standard form of length by height, assuming the principle that this concept corresponds with the natural passing of square units held in the quadrangle, so they register areas as: $\mathrm{T}^{2}=\mathrm{TxT}$. For its measuring they built an arithmetic geometrical tool: The Longest Quadrilateral which allowed them to check the quadrilaterals of each plot, and with this they made possible to break them into elemental figures: parallelogram, triangles and squares, and with this it was possible to estimate their maximum area, contrasting it with the registered area, which is supposedly shown in the plots. They say that the most used algorithm by acolhuas to determine the area was the surveyor rule a Sumerian method that focuses in the 
technique of multiplying the averages of opposing sides. Inside the registers that appear in the codex, there are cases in which the plots were located including in each side their respective perimeter lengths, in units $\mathrm{T}$ and, under these, the same plots with an inscription in the lower part of the center, that indicates a length, also in units $\mathrm{T}$, as well as a $t a b$ in the upper right margin that shows lineal units, Figure 1: the tab is the place of units. It is an adequate positional system of acolhuas to register their areas. There is evidence of the existence of a measure of land that was equivalent to $20 \times 20$, from there the $\mathrm{T}^{2}(2)$. According to the authors, to figure out the plots area under these conditions, it is enough to take the symbolic values that are in the version of the plots in the lower part, to add after the decimal point the quantity that appears in the tab, and multiply it by 20 . Under that assumption the area of the first plot contains the information: $\bullet=20 \mathrm{~T}$, 1 i $=5 \mathrm{~T} \mathrm{y} \mid=\mathrm{T}$, that in a set it must be:

○周而而 ||

As a result, adding after the point the quantity that appears in the tab:

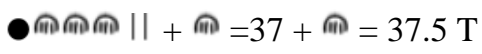

So, the area registered on the plot results to be:

$$
\begin{aligned}
& 37.5 \times \bullet=750 \mathrm{~T}^{2} \\
& 37.5 \times 20=750 \mathrm{~T}^{2}
\end{aligned}
$$

The numerical system that is appreciated in the measures of the lands done by M. C. Jorge workgroup, becomes an anthropocentric model that determines a universe of numbers that are playing a role, this is evidenced by other researchers [2], [3] and [4]. Under this point of view, this must be seen as an approach model in which mistakes are on the center of the main numerical set. Lacking ethnographic sources that show a definition of a better summary of measure and numerical systems, authors determined the monads, fragments of a measuring instrument that was used based in the vigesimal system and the limbs of human body. A heart or yollotl, symbolized as is $2 \div 5 \mathrm{~T}$, a hand $\mathrm{m}$ is $3 \div 5 \mathrm{~T}$ and a bone is $1 \div 5 \mathrm{~T}$. That is where relations with other fragments are obtained.

$2 \mathrm{x}$ arrow $=5 \mathrm{x}$ bone $=1 \mathrm{~T}, 5 \mathrm{x} 1$ yollotl $=2 \mathrm{~T}, 5 \mathrm{x}$ hand $=3 \mathrm{~T}$, and it is obtained $1,2,3$, in a certain way, they give a sense to the fractions whose multiples must be entire numbers of the unit.

As a consequence of this view, the numerical and measuring systems are seen without uniformity between the main cultures that are part of Mesoamerican civilization, as if each group had adopted different values in the measurements of the mentioned fragments. To understand the uses this cultures made of measurement systems it is necessary to go to the main origin of such systems. In the next section two fundamental issues are raised which give evidence of the evolution in them. One of them is the magnitudes in feet and yollotls the block of stone the Aztec goddess Tlaltecuhtli, found in 2006 in the Major Temple, located in Historical Center, México City, as well as the Bas-relief of Xoc, elaborated by Olmec culture about 1,600 B. C., found in Ocosingo Chiapas, around the sixties of last century. The second view taken in this article, in which the definition of both systems is sustained from the Synodic and Sidereal revolutions of planets that Mesoamerican astronomers commonly observed. The important part of this research is focused in explaining the mathematical and astronomical content of the three plots previously mentioned, Figure 1, included in the Santa María la Asunción y Vergara codex. For its analysis, it is started with a multidisciplinary vision that involves faculties, which are similar to the ones used by Mesoamerican ethnics for the rising of their monuments, in this case surveying, astronomy and mathematics among others.

\subsection{Magnitudes of the Tlaltecuhtli}

The block of rectangular sculpture of the Tlaltecuhtli was accidentally discovered in October 2006 on the base line of one stairway of Mayor Temple, which was broken into four big pieces. Due to the risk, its extraction lasted about a year, so an interdisciplinary research team was organized that, together with a group of experts of INAH and UNAM (3), dealt with restoring as well as analyzing under different perspectives the Materiality of the Monument [5]. The scientists identified the porphyritic type of the stone, its structure, longitudinal measurements, the pigments with which the image was painted among other important issues that leaded to its design and elaboration. 

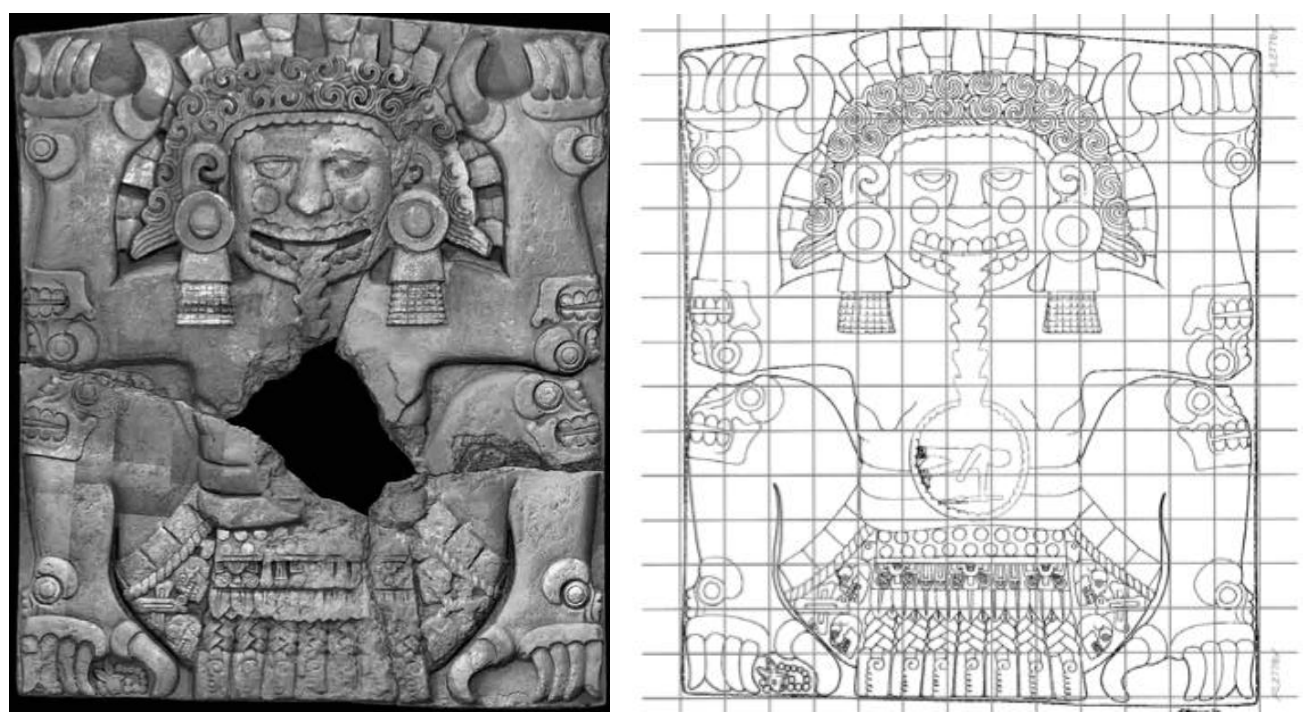

Figure 2. Rectangular mesh over the block, designed from the unit of measurement known as yollotli, or heart, of $0.8334 \mathrm{~m}$ Source: [5].

Once the pieces were matched, left image Figure 2, it was possible to precisely establish the original measurements of the block, there are $417 \mathrm{~cm}$ in the vertical part by 362 in horizontal part and 25 to $37 \mathrm{~cm}$ depth, which were determined out with a tridimensional scanner with which over 50,000 measurements per second were reached through a computer. Under the assumption of its length, researchers believe that the measurement units used for the designing and elaboration of the block are in yollotls (from Nahuatl, heart) that are known as Mexica measurement units. In fact, the measurement in meters of a yollotl is close to $0.8334 \mathrm{~m}$.

These values make sense when they are divided between two of the main patterns used by Mexica people: the yollotli or heart and the tlacxitamachihualoni or foot. The first is close to a Castilian stick that equals exactly to $83.34 \mathrm{~cm}$, while the second is the third part of a yollotli, it means, $27.78 \mathrm{~cm}$ (Ibíd. pp. 53-56). This point of view assumes the existence of a numerical system that was used by groups on central Mexico to perform longitudinal measurements, mainly in the elaboration of monuments. In this case the yollotl of $0.8334 \mathrm{~m}$ is mentioned as well as the tlacxitamachihualoni, or foot, of $27.78 \mathrm{~m}$. Researchers drew a grid over the image of the goddess; right part Figure 2, that in its extension shows symmetry in its design, and also the accuracy with which it was measured through the previously mentioned measurement units. From the mesh they obtained a correspondence of 4.334 yollotls, 13 feet, for the short side of $362 \mathrm{~cm}$, and 5 yollotls, 15 feet, for the long side of $417 \mathrm{~cm}$.

However, the fragments of yollotl and foot are truly regulated by incommensurable rational numbers, in this case: $83.333 \ldots \mathrm{cm}$, for the first, by $27.777 \ldots \mathrm{cm}$, for the second, that give more sense and accuracy to the measurement tool known as Tlalquahuitl, that in Nahuatl means Pattern and corresponds to the unit T, that its size is $2.5 \mathrm{~m}$. From this point of view, the quotient of the sides of the Tlaltecuhtli block, measured in feet, sets the first constant between them, this is: $1.153846 \ldots=15 \div 13$. Which reciprocal represents at the same time, another constant of the same nature, this is: $0.8666 \ldots=13 \div 15$.

In the following pages, both are called fundamental astronomical constants; the ones that frequently appear in the monumental magnitudes and let to a yield in astronomical environment lived in Mesoamerica.

\section{ORIGIN OF NUMERICAL AND MEASUREMENTS SYSTEMS}

\subsection{First correlations}

At the end of XIX century, German researcher E. Förstemann [6] evidenced a calendar arithmetically coded from an analysis of a portion of the one known as Dresde Codex that is believed to be the hieroglyphic Maya system. In this he discovered correlations between the synodic and sidereal movements, both the moon, the earth, and Venus and Mars. And also, he estimated the movements of Venus and the Earth from their average values, such as 584 and 365 days, thus considering a fundamental correlation between them, as:

$$
\mathbf{2 , 9 2 0}=584 \text { × } 4=365 \times 5 \text { days }
$$

In the same path, according to the El Economista newspaper version, the Xoc Bas-relief was performed by the Olmec culture about 1,600 years B.C., which was taken out of the country more than 40 years ago, and now it has been recovered. The piece was seriously damaged causing unfixable harm to the monument and it represents a tremendous anthropological importance, because: 
(...) a proof of the presence of this affiliation human groups out of the nuclear area of this culture, set on the South of Veracruz and part of Tabasco, coastal states of the Gulf of Mexico. Which its 2.20 meters tall and 1.15 meters width and a 30 centimeters depth, it represents a standing man, in profile with symbolic clothing, that is wearing a mouth mask, a high headwear and who has feet in the form of claws, which are typical features of Olmec iconography.

http://eleconomista.com.mx/entretenimiento/2015/09/24/bajorrelieve-olmeca-xoc-regresa-mexico-tras-surobo.

A first model of translation of magnitudes is presented in centimeters on the rectangle that contains the Xoc Bass-relief, to fragment units that are taken out of unit $\mathrm{T}$, in this case yollotls. To make this easier the length results given by El Economista have been kept, that is $220 \mathrm{~cm}$ in $219.375 \mathrm{~cm}$ for the length, subtracting 6.25 $\mathrm{mm}$, also to the length of the width, of $115 \mathrm{~cm}$, there have been added $2 \mathrm{~mm}$, leaving it as 115.2 (4).

The point of view that is drawn with this is that both lengths, corrected and translated to yollotls become astronomical magnitudes. In this case the procedure that leads to the translation is the product:

$$
\text { Magnitude in } \mathrm{cm} \times 0.8333 \ldots \mathrm{m}=\text { Astronomical Magnitude in days (5) }
$$

The results bring the magnitudes nature of origin with which the Bass-relief was made. The magnitudes translation to yollotls appears as:

$$
\begin{gathered}
219.375 \times 0.8333 \ldots=\mathbf{1 8 2 . 8 1 2 5} \text { days } \\
115.2 \times 0.8333 \ldots=96 \text { days } \\
30 \times 0.8333 \ldots=25 \text { days }
\end{gathered}
$$

The translation makes clear that the long length of: $219.375 \mathrm{~cm}$, corresponds to a half solar year of: $182.8125 \mathrm{~T}$, that must be taken in days, because: $365.625 \div 2=182.8125$ days (The Mesoamerican solar year being 365.625 days, as shown below). In the same way, the $115.2 \mathrm{~cm}$ are equivalent to 96 days, corresponding 25 days to $30 \mathrm{~cm}$ of depth.

Observe that the product of the three magnitudes:

$$
182.8125 \times 96 \times 25=438,750 \text { days, }
$$

correlates the movements of Venus (585 days) and Mars (780 days) as well as the ritual (260 days) and solar years (365.625), as of the highlighted numbers:

$$
438,750=\mathbf{5 8 5} \times 750=\mathbf{3 6 5 . 6 2 5} \times 1,200=\mathbf{7 8 0} \times 562.5=\mathbf{2 2 5} \times 1,950
$$

These last expressions show that the design and construction of the Bass-relief, was because they wanted to put a solar calendar over its materiality that allowed them to control the involved planets movement. In addition, it gives an evidence of the number that determines the solar year of 365.625, the Venus movements of 585 and 225 days, as well as the synodic movement of Mars that is 780 days, without excluding the ritual year of 260 days.

Through a correlation, it is easy to prove that the regularities on the synodic movements of Venus, regarding the Earth movement, draw from the fact that the conjunctions between them are produced every 2,925 days, different in 5 days to Föstermann's view. Dividing the previous correlation between 150 days and leaving only the Venus synodic movement and the solar year it is:

$$
\mathbf{5 8 5} \times 5=2,925=\mathbf{3 6 5 . 6 2 5} \times 8
$$

It turns out that 5 periods of 585 days of Venus synodic cycle, equal at the same time to 8 solar years of 365.625 days. After all this time it occurs a Venus conjunction with the Earth being in the same place that is was 8 years before, it means, an alignment of both planets regarding the Sun. This points out that 8 Venusian years are equivalent to 5 years in Earth. In the same correlation appears the Venus sidereal cycle, renowned as 225 days that can be showed as: $2,025=225 \times 13$.

These correlations set two first cardinal astronomical constants that can be accommodated under the ratios:

$$
\text { 2.6 }=13 \div 5=585 \div 225 \text { and } \mathbf{1 . 6}=8 \div 5=585 \div 365.625
$$

From the 2.6 constant derives the ritual year of 260 days, which was of great help among Mesoamerican ethnic groups. Even the proportion between solar and ritual years determines the, also astronomical constant:

$$
\mathbf{1 . 4 0 6 2 5}=365.625 \div 260
$$

With the same constant 2.6, a parallel between the synodic cycles of 780 years of Mars is established, with regard to 300 days that are taken from its sidereal movement, this is:

$$
2.6=780 \div 300
$$

For the Moon, its synodic and sidereal cycles are have been placed on the monuments and pyramids-temples as 29.5 and $27.23077 \ldots$ days, which establish the constant 1.08333, because:

$$
1.08333 \ldots=29.5 \div 27.23077 \ldots
$$


The definition of the Mesoamerican planetary system is drawn from considering that the year is: $365+$ $(8 \div 5)=365.625$ days, that is subject to the system determined by numbers 5,8 and 13 , that are shown in the correlation. From the quantity $8 \div 5$ and the solar year, Venus synodic movement is deducted, which result in the product: $365.625 \times(8 \div 5)=585$ days.

Even the synodic product of 585 days done by computer 13, determines what it is known as angular movement of the planet developing along its own synod that appears in days, this is:

$$
585 \times 13=7,605 \text { days }
$$

The angular movement appears in the same way to the rest of the planets. This principle is only settled without any explanation. The result: $365.625 \times(8 \div 5)=585$ days, shows certain arithmetical rigor in correspondence with the synodic revolution of Venus regarding the solar year, that establishes a first link of the astronomical system. The fact that the solar year has been considered in Mesoamerica as: 365.625 days and not in the approach as it is nowadays recognized of 365.25 days, it does not mean an astronomers ignorance of this fact, but that this quantity corresponds to the numerical system structure organized with that purpose. The astronomical sizes: 585, 225, 365.625, 780, 300, 29.5 and $27.23077 \ldots$ have been built along centuries of accurate observations that conducted to their establishment. Even the power of these correlations articulates them in their entirety, so none of them is at random, that is:

$$
585 \times 5=2,925=365.625 \times 8=225 \times 13=780 \times 3.75=260 \times 11.25
$$

Numbers: $5,8,13,3.75$ y 11.25 are parte of the numerical system that is shown in the correlation.

\subsection{Twentieth-thirtieth system}

As it is seen, the correspondence of proportions: $(13 \div 5)=(585 \div 225)$ and $(8 \div 5)=(585 \div 365.625)$ is the key to understand the relation between numbers 5,8 and 13 with the planets movements. In this sense, these numbers are the ones that led to the creations of this numerical system, which has been called twentieth-thirtieth, because it is generated by the previously mentioned numbers and 20, that determines the vigesimal Maya. Observe how these numbers appear in the proportion of Mars movements:

$$
780 \div 300=(780 \div 13) \div(300 \div 13)=(60=20 \times 3) \div(300 \div 13)=\mathbf{2 . 6}
$$

The first unit of measurement of this system, probably the fundamental, was determined by the astronomical constant 2.6 divided by 3 , in the form:

$$
2.6 \div 3=13 \div 15=0.8666 \ldots
$$

With the last, it was possible to attract the astronomical universe and set it in the building of the first pyramidtemples. As such, the one of Sun Temple in Teotihuacan, has a magnitude of 86.666... T, 216.666... m (6), per side that is found making the division of synod Mars movement of 780 days, between 9; or by the ritual year of 260 days, such as:

$$
780 \div 9=260 \div 3=86.666 \ldots
$$

On the same way, the length of Quetzalcoatl Temple square base, in the same place is $26 \mathrm{~T}, 65 \mathrm{~m}$, in an analogy with the astronomical constant 2.6 previously mentioned.

Other important constants due to their wide use in monuments, are the ones calculated with the reciprocal calculation $0.8666 \ldots$ mentioned before:

$$
3 \div 2.6=15 \div 13=1.153846 \ldots
$$

as well as doubling the first: $2 \times 0.8666 \ldots=1.7333 \ldots$ However, the measurement system is incomplete if it lacks a principal piece: the measurement tool, How to measure on the plot these $86.666 \ldots$ units of the pyramid side, so then they can start its construction?

Just as the French scientists attracted the metro-pattern of the ten million part of the quarter of a terrestrial meridian, Mesoamerican wise men attracted the first fragment of the measurement tool of the main center of the planetary universe. The calculation to get it was to divide the astronomical constants, $0.8666 \ldots$ and 2.6 as:

$$
0.8666 \ldots \div 2.6=0.333 \ldots
$$

Because the fragment is $0.333 \ldots$ it was attracted from the main heart of the planetary movement, surely they called this yollotl, that in Nahuatl it means heart. Then they fragmented the yollotl, under a unified regulation rule, the first calculus was to divide it by 3 that led to:

$$
0.333 \ldots \div 3=0.111 \ldots
$$

that corresponds to a segment of the first, which was called tlacxitamachihualoni or foot. Subsequently, multiplied by three it is:

$$
3 \times 0.333 \ldots=1
$$

The complete unit instituted Tlalquahuitl, as an essence to the measurement tool with its own segments, ready to 
use it to measure the needed sizes in units $T$.

Since in $\mathrm{T}$ units the yollotl definition is determined as a rational number, multiple of the organizer 13 , in the form: $0.333 \ldots$ as well as the foot in a number of the same nature, as $0.111 \ldots$ these quantities in meters are:

$$
0.3333 \ldots \times 2.5=0.8333 \ldots
$$

to the yollotl and:

to the foot.

$$
0.111 \ldots \times 2.5=0.2777 \ldots
$$

In the three measurement fragments the numbers correspond to rational numbers, commensurable and incommensurable, they all of the rational nature. The set of numbers groups some operations derived from the twentieth-thirtieth system, which makes sense by the definition of Tlalquahuitl fragments. Observe that it is no possible to determine the foot in $0.2778 \mathrm{~cm}$, for a very simple reason that has to be with 13 and 15 foot this is how the grid, overlapped to the Tlaltecuhtli, is divided. In short, if we accept that magnitude, the constant: $15 \div$ $13=1.153846,153846 \ldots$ that immediately emerges in most monument magnitudes is not verified with that accuracy. In this way, the symbols that are associated with the previously mentioned lengths, are the scores:

that symbolize the $20 \mathrm{~T}$ magnitude, and its integral multiples: the hands $\mathrm{E}$ - $\mathrm{T}$, and the fingers or stripes $।, 1 \mathrm{~T}$, as well as the fractions scaled by the organizer 13: the yollotl, symbolized in the shape of a heart of $0.333 \ldots \mathrm{T}$ and its multiples, as the foot, $\rightarrow$ that as it was mentioned, it is: $0.111 \ldots \mathrm{T}$. The arrows, $0.5 \mathrm{~T}$, as well as

elbows or bones $\mathbb{R}, 0.2 \mathrm{~T}$, are vigesimal sub-multiples.

The next example they are shown under the same numeric accumulation, it is: $37.333 \ldots \mathrm{T}$ :

$$
\text { 부붕 }
$$

number in which the symbols have been set ordering them from left to right, placing the twenties first, black circles $\bullet$, after them, multiples of 20 are set, it is the hand case $\mathbb{E}^{\mathbb{E}}$, as well as the stripes I, or fingers, putting the fractions at the end, in this case a yollotl . Observe that the last fraction makes the decimal point to float, making it ostensible, with no need to symbolize it. That makes the zero position not visible either. The previous sequence shows that the integral values of $\mathrm{T}$, in most cases, can be multiples of the organizer 13 or the computer 20 , even positive integral numbers, while the fractions can have multiplicities of one or the other, also including integral multiples. Unlike the Mesoamerican, the metric system is folded over a totally decimal system, in which setting the magnitude orders, and respecting the decimal positions, requires a single calculation. In the twentieth-thirtieth system these operations are not entirely because they do not pay attention to the position of the decimal point and the zero existence, putting it aside from the Maya vigesimal. However, its accumulative and symbolic nature, eliminates that problem and makes it very similar to the decimal system, putting it as a dynamic system easily understandable and useful, which numbers come from the possible combinations of the positive integrals, and the organizer 13 and computer 20. In this sense, the numeric universe is a subset of rational numbers that is symbolized as $\mathrm{Q}$, from which the negative integrals are simply taken out, while the zero and the decimal point are not visible, more ostensible. However, behind the twentieth-thirtieth system the real measurement numerical system is hidden, this comes from the planet movement and it was used in the projecting and building of every kind of monuments, as it has been shown in the translation of magnitudes of the Xoc Bas-relief. The symbolic numeric model presented this far, stands out from the time in which groups of the Fifth Triple Alliance Mexica were in their most cultural and scientific effervescence, close to the years 1,400 to 1,450 A.D until the conquest. The technical arguments presented before, are fundamental to explain the metrological content that occurs in the irregular plots of the research made by M. C. Jorge work team.

\subsection{First plot}

\section{IRREGULAR PLOTS IN THE CODEX}

The main problem that acolhuas engineers must face to the geometrical and astronomic design of the plots was to accurately define the magnitudes of their sides, so they were consistent with their own area that can be pre-determined. In the three plots the area is different, because each of them was made to represent a different astronomical model. An explanation of each is given down below. In the image shown in Figure 3, magnitudes of the perimeter sides of the first plot are included in $\mathrm{T}$ units, on the left, and about complementary information of it, that it is not necessary assumed to the area, in the right one. The reader can verify that these last correspond with the symbolic values of the images shown in Figure 1. For this first plot an analysis will be done from the magnitudes in meters and $\mathrm{T}$ units, luckily, the reader will be able to measure the astronomical magnitudes resulting in each case. It must be clear that the use of units in meters represents a simple change of scale in the design and building of monuments. In this respect, it can be verified that the change of scale results to be $1 \mathrm{~m}=0.4 \mathrm{~T}$. For the first plot case, it is enough to multiply by 2.5 each magnitude, to determine the corresponding lengths in meters. In both systems the four lengths are: 


\section{$24 \mathrm{~T} / 60 \mathrm{~m}, 20 \mathrm{~T} / 50 \mathrm{~m}, 32 \mathrm{~T} / 80 \mathrm{~m}$ and $30.333 \ldots \mathrm{T} / 75.8333 \ldots \mathrm{m}$}

If then the surveyor rule were used to determine the area, we would have the groups of average values in both scales. In meters it would be:

$$
(75.8333 \ldots+80) \div 2=77.91666 \ldots \text { close to } 78 \text {, and }(50+60) \div 2=55
$$

The first has been rounded off to $78 \mathrm{~m}$, leaving the second as it is, so the area results to be:

$$
78 \times 55=4,290 \mathrm{~m}^{2} / \text { days }
$$

This makes a cluster of correlations between Venus, Mars and ritual and solar years movements as this:

$$
4,290=\mathbf{5 8 5} \times 7.333 \ldots=\mathbf{2 2 5} \times 7.333 \ldots \times 2.6=\mathbf{3 6 5 . 6 2 5} \times 7.333 \ldots \times 1.6=\mathbf{2 6 0} \times 7.333 \ldots \times 2.25=\mathbf{7 8 0} \times 5.5
$$

The area in $\mathrm{T}$ units corresponds with the one of $4,290 \mathrm{~m}^{2} /$ days, dividing each length by 2.5 , these are: $31.2 \times 22$ $\mathrm{T}$, which determine an area of:

Correlations given by this last one are:

$$
31.2 \times 22=686.4 \text { days }
$$

$$
\begin{gathered}
686.4=\mathbf{5 8 5} \times 1.17333 \ldots=\mathbf{2 2 5} \times 1.17333 \ldots \times 2.6=\mathbf{3 6 5 . 6 2 5} \times 1.17333 \ldots \times 1.6=\mathbf{2 6 0} \times 1.17333 \ldots \times 2.25= \\
=780 \div 1.136363 \ldots=300 \times 1.17333 \ldots \times 1.95
\end{gathered}
$$
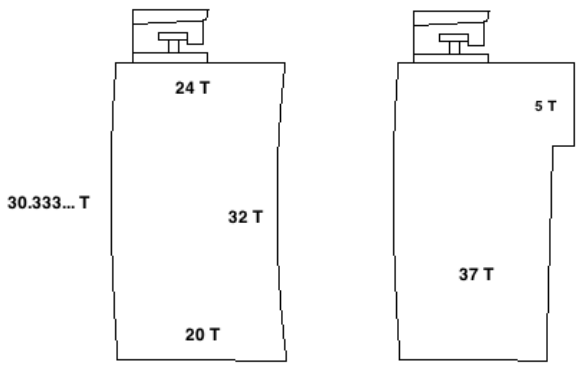

Figure 3. Left, perimeter information of the first plot and complementary magnitudes in the right. Compare with Figure 1. The plots do not have a scale.

In the correlation the Mars med movement of 300 days has been included, that together with the synodic movement determine the astronomical constant 2.6 , that is: $2.6=780 \div 300$. However the sidereal and synodic movements of the same planet, are shown in the correlation as those of 780 days and 686.4 days, and they establish the astronomical constant:

$$
780 \div 686.4=1.136363 \ldots
$$

This means that the area of the first plot corresponds to Mars sidereal movement established by acolhuas astronomers in 686.4 days. The current value of the sidereal movement comes up from 686.971 days, https://es.wikipedia.org/wiki/Marte_(planeta) and the synodic movement is shown as: 779.96 days. In such a way that the proportion resulting with them is:

$$
779.96 \div 686.91=1.1354617 \ldots \text { days }
$$

The differences, in both synodic and sidereal movements, as well as the proportion between the same magnitudes, which were given recently, and the ones used by Mesoamerican cultures, are these respectively:

$$
\begin{aligned}
& 780-779.96=0.04 \text { days, } \\
& 686.971-686.4=0.571 \text { days, and } \\
& 1.136363 \ldots-1.1354617 \ldots=0.009013 \text { days }
\end{aligned}
$$

For their part, the information that appears in the second image on Figure 3, shows number $37 \mathrm{~T}$ in the rectangle center, and 5 in the upper tab. Observe next that both, the area and the two numbers, have multiplicities in the astronomical constants $1.153846 \ldots$ and $1.08333 \ldots$ that is:

$$
\begin{gathered}
686.4=1.153846 \ldots \times 1.08333 \ldots \times 549.12 \\
37=1.153846 \ldots \times 1.08333 \ldots \times 29.6 \\
5=1.153846 \ldots \times 1.08333 \ldots \times 4
\end{gathered}
$$

It is: $1.153846 \ldots \times 1.08333 \ldots=\mathbf{1 . 2 5}$. From here it results a triple proportion between the involved magnitudes, that it is possible to put it as:

$$
686.4 \div 549.12=37 \div 29.6=5 \div 4=\mathbf{1 . 2 5}=1.153846 \ldots \times 1.08333 \ldots
$$

This triple proportion guarantees to have other alternatives of building new constructions or monuments on the ground, compliant with the involved magnitudes of length and area, in such a way that they correlate with other possibilities of the planets movements. Thus, for example, magnitudes of area and length of the plot can be 
given with a double solar year of: $731.25=2 \times 365.625$, and Venus synod, or with the ritual year and doubling the magnitude $104(10.4=2.6 \times 4)$ Scheme 1 , as:

$$
\begin{aligned}
& (686.4 \div 549.12)=(37 \div 29.6=5 \div 4=\mathbf{1 . 2 5})= \\
& \text { Magnitudes of the land, Magnitudes of the twentieth-thirtieth system, Astronomical magnitudes }
\end{aligned}
$$

Scheme 1. Transition between local magnitudes, left, twentieth-thirtieth system magnitudes, center, and astronomical magnitudes, right.

Observe that magnitudes 37 and 5 set in the tab on the second image of Scheme 1, are part of the twentieththirtieth system and they are also transitional magnitudes between the main magnitudes imposed to the plots and those that let astronomical fate. This results into accurate correlations between the planets movements and ritual and solar years. In the case that involves Mars sidereal cycle we have:

$$
\mathbf{1 . 2 5}=686.4 \div 549.12=260 \div(104 \times 2)
$$

The result is:

$$
142,771.2=686.4 \times 208=260 \times 549.2
$$

Which it can be paraphrased as: Mars does 208 sidereal revolutions of 686.4 days in 549.2 ritual years of 260 days each. This happens in 142,771.2 days.

\subsection{Second plot}

The magnitudes averages in units $\mathrm{T}$, of the second plot sides, Figure 4, are 58.75 and $69.1666 \ldots$. Which have been rounded off to $59 \mathrm{~T}$ and $69.03 \mathrm{~T}$ :

$(57.5+60) \div 2=58.75$ close to $\mathbf{5 9}$ and $(58.333 \ldots+80) \div 2=69.1666 \ldots$ close to $\mathbf{6 9 . 0 3}$
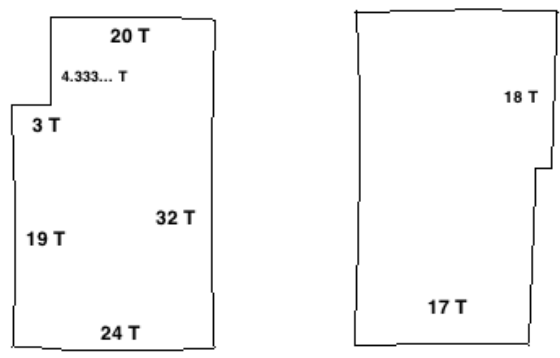

Figure 4. Left, perimeter information of the second plot, and complementary magnitudes on the right. The plots do not have scale.

Both sides are in proportion as: $69.03 \div 59=1.17=0.585 \times 2$. The proportion establishes a correlation between the Moon and Venus movements that is shown in the following:

$$
17,257.5=585 \times \mathbf{2 9 . 5}=633.75 \times \mathbf{2 7 . 2 3 0 7 7} \ldots
$$

There is even a correlation with Venus sidereal movement of 225 days, from its side it appears an important constant of the Moon movement, and this is 76.7 days.

$$
17,257.5=585 \times \mathbf{2 9 . 5}=225 \times \mathbf{7 6 . 7}
$$

However, the correlation can be broader, because it also involves the satellite angular movements, synodic and sidereal of 383.5 and 354 , without leaving the correlation with ritual and solar years aside, that is:

$$
17,257.5=585 \times \mathbf{2 9 . 5}=225 \times \mathbf{7 6 . 7}=\mathbf{3 8 3 . 5} \times 45=\mathbf{3 5 4} \times 48.75=\mathbf{3 6 5 . 6 2 5} \times 47.2=\mathbf{2 6 0} \times 66.375
$$

Observe that the angular movements condition synodic and sidereal movements. The angular movements are assumed to the same condition imposed to the same Venus angular movement, previously mentioned, it is: 383.5 $\div 13=29.5$ days and $354 \div 13=27.23077 \ldots$.

Even if the reader cannot see it immediately, the magnitude 47.2, associated with the solar year in the correlation, it is also a cardinal constant that organizes the Moon movements. In fact, this last and the one of 76.7 days, come from the Moon synodic movement of 29.5 days that is associated with other fundamental constants. Observe this quality:

$$
\begin{aligned}
& 76.7=\mathbf{2 9 . 5} \times 2.6 \\
& 47.2=\mathbf{2 9 . 5} \times 1.6
\end{aligned}
$$

The proportion between these makes the resulting fundamental constants to correspond because:

$$
76.7 \div 47.2=1.625=\mathbf{2 . 6} \div \mathbf{1 . 6}
$$

The constant 1.625 days, induces to establish other correlation, not less important, between the solar year and Venus sidereal cycle, which is: 


$$
76.7 \div 47.2=1.625=(585 \div 225=\mathbf{2 . 6}) \div(585 \div 365.625=\mathbf{1 . 6})=(\mathbf{3 6 5 . 6 2 5} \div \mathbf{2 2 5})
$$

That let us see the importance of a huge amount of correlations emergence, which are needed to recognize the planetary movements, that have the solar and ritual years as a reference for the count. Also, magnitude 66.375 days turns out as one side of Square of the Moon in Teotihuacan, that in this case it appears as a simple counter.

From the side of the plot area, this results: $50 \times 69.03=4,072.77$, that correlates with the Moon movements, in the form:

$$
50 \times 69.03=4,072.77=\mathbf{3 8 3 . 5} \times 2.95 \times 3.6=\mathbf{3 5 4} \times 2.95 \times 3.9
$$

Regarding numbers 18 and 17 that are shown, first on the side note, and the other in the tab second image, Figure 4, they can be written as:

$$
18=5 \times 3.6
$$

$$
17=(85 \div 18) \times \mathbf{3 . 6} \text { as well as } 18=(85 \div 17) \times \mathbf{3 . 6}
$$

Even the previous correlation: $4,072.77=\mathbf{3 8 3 . 5} \times 2.95 \times \mathbf{3 . 6}$. The clue that links not only the area but also numbers 18 and 17 is the constant 3.6. From numbers 18 and 17 we have: $3.6=(18 \times 17) \div 85$. Replacing this last in the place that corresponds to it the correlation is:

$$
4,072.77=\mathbf{3 8 3 . 5} \times 2.95 \times(18 \times 17) \div 85
$$

From which we obtain the product:

$$
346,185.45=\mathbf{3 8 3 . 5} \times 2.95 \times 18 \times 17
$$

This last correlation with the Moon and Venus movements, and the solar year as well, it is as follows:

$$
\begin{gathered}
346,185.45=585 \times 383.5 \times 0.2723077 \ldots \times 5.666 \ldots \\
346,185.45=365.625 \times \mathbf{3 5 4} \times 0.295 \times 5.666 \ldots \times 1.6 \\
346,185.45=225 \times \mathbf{3 5 4} \times 5.666 \ldots \times 0.767
\end{gathered}
$$

From these correlations the usefulness and importance of magnitudes 18 and 17 are estimated, they are inserted in the rectangle that is visible on the right side of Figure 4. It is obvious that these last are not accidental and it can be assumed that they do not have any relation with the plot area that is shown in the upper part, but with the astronomical condition that occurs in it.

\subsection{Third plot}

The averages on the side's magnitudes of the plot are $33.5 \mathrm{~T}$, for length, by $19.645 \mathrm{~T}$, width, Figure 5 . The area provided by both results to be:

$$
33.5 \times 19.645=658.125 \mathrm{~T}^{2}
$$

This correlates with the solar year and Venus movements as:

$$
658.125=585 \times 1.125=225 \times 2.925=225 \times 2.6 \times 1.125=365.625 \times 1.6 \times 1.125
$$

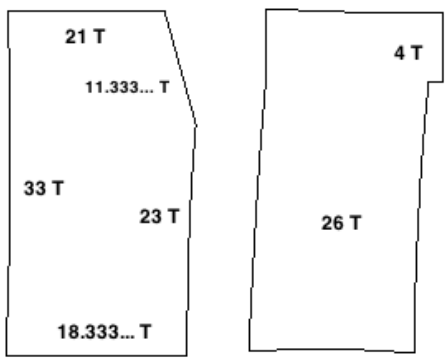

Figure 5. Left, perimeter information of the third plot and complementary magnitudes on the right, center and the tab. The plots do not have scale.

Doing the division between the magnitudes set on the plot on the right, shown in Figure 5, it is: $26 \div 4=$ 6.5. If then we multiply this last with the area in days: 658.125 , of the plot on the left it results: $6.5 \times 658.125=$ 4,277.8125 days. Which fully correlates with Venus and Mars movements, when ritual and solar years helped to make the calculation, this is:

$$
4,277.8125=365.625 \times 58.5 \times 2=585 \times 7.3125=225 \times 10.0125=260 \times 3.65625 \times 4.5=780 \times 3.65625 \times 1.5 \text {. }
$$

Similar to Scheme 1, in Scheme 2, we show the intercalation between magnitudes that occur in the plot, in this case in the third plot, the magnitudes of transit on the twentieth-thirtieth system and those astronomical magnitudes of the planetary movement that are given with magnitude 6.5 that is shown in the tab of right image in Figure 5.

$$
\begin{aligned}
(658.125 \div 101.25)=(26 \div 4=\mathbf{6 . 5})=\quad & (365.625 \div 56.25=585 \div 90)= \\
& =(780 \div 120)=(260 \div 40)
\end{aligned}
$$




\section{Magnitudes of the land, Magnitudes of the twentieth-thirtieth system, Astronomical magnitudes}

Scheme 2. Transition between local magnitudes, magnitudes from the twentieth-thirtieth system and astronomic magnitudes.

It is evident that the magnitudes of each of the three lots represent planetary movements. The first plot was done to keep a control in Venus travels, starting from the sidereal cycle of Mars based over the area of the same plot, in which solar and ritual years serve to keep counts. The second plot is a container of astronomical information of the Moon movements correlated with those of Venus and Mars, helping to make the counts, solar and ritual years. Finally the third plot was made to keep a control of the movements of Venus, under the same circumstance of the solar and ritual years. Furthermore, the plots that are under the ones that were analyzed, show numbers of twentieth-thirtieth system that establish a transition between magnitudes contained in the plots located at the top and the planets movements. This quality is even current in all the grounds that are connected with Santa Maria la Asuncion y Vergara codex.

\section{CONCLUSION}

It can be considered that length and area magnitudes of the analyzed plots are located in a period in which acolhuas surveyors and astronomers downloaded the astronomic magnitudes that were suitable to the monuments designing and building, to do the cadastral metrological order. But this does not ensure its existence on the ground. These last determine a symbolic model representing the astronomical reality known by the Mesoamerican group, in sum, a cartography that also shows an important evolution in the numeric establishment of some planet cycles as in the case of 686.4 days, on the sidereal period of Mars, as well as the involved astronomical constants. Observe that the area in meters 4,290 of the First Plot, divides the same area of 686.4 in $\mathrm{T}$ units, establishing in an equivalent way the astronomical constant 1.6 that provide the synodic movement of Venus with the solar year that occurs as follows:

$$
6,864 \div 4,290=1.6=585 \div 365.625
$$

This leads to correlate the involved planetary movements.

On the same path, the researchers group previously mentioned, established the area of plot known as El Topote, which was already mentioned in the introduction, estimated in $124,071.52 \mathrm{~m}^{2}$. A point of view is soon assumed in which it is considered that the original astronomical area was: $123,581.25 \mathrm{~m}^{2}$, with an error margin regarding the previous of $490.27 \mathrm{~m}^{2}$. Even that it is measured in meters, the area conceived as this, correlates with all planetary movements, including the solar an ritual years because:

$$
\begin{gathered}
123,581.25=365.625 \times 338=225 \times 169 \times 1.153846 \ldots \times 1.08333 \ldots \times 2.6=585 \times 169 \times 1.153846 \ldots \times \\
1.08333 \ldots=686.4 \times 180.042613=300 \times 169 \times 2.4375=780 \times 65 \times 2.4375=260 \times 195 \times 2.4375
\end{gathered}
$$

This research shows a stable numeric universe, as well as concepts of area and proportion, which are useful in establishing correlations. These concepts and the arithmetical calculations that go together with them, are completely built about 1,600 years B.C, as evidenced by the magnitudes of the Bas-relief of Xoc, which indicates that the processes that leaded to its building must happen centuries ago. Inclusively the numbers that represent planetary movements, as well as those of solar and ritual years, occur in the broad period from 1,600 years B.C to 1,500 years A.C. Finally, the interdisciplinary focus employed to the analysis of the three plots on the codex, have enabled to offer a better interpretation of its content, that encourages the research of similar ones, including monuments raised by groups along centuries.

\section{ACKNOWLEDGEMENTS}

To Danièle Dehouve (Anthropologist) Centre National de Recherche Scientifique (C.N.R.S) France, by the preliminary lecture of the manuscript.

To Patricia Villanueva (Licensed in English language) Engineering Faculty of Autonomous University of Chihuahua, Mexico, for translating the manuscript into English.

(1) In the next pages it will be simply M.C. Jorge.

(2) This quote and the next come from e-mail contact between the author and Ph. María del Carmen Jorge, during 2015.

(3) I.N.A.H, Instituto Nacional de Antropología e Historia. U.N.A.M, Universidad Nacional Autónoma de México.

(4) Observe that the ones, who report the Bass-relief magnitudes in El Economista, were not measured with millimeter approximation. From there, the measuring tape used was only close in centimeters, which distorts the yollotls translation. 
(5) The quotient can be verified: Magnitude in $\mathrm{cm} / 0.8333 \ldots$ also determines astronomical magnitudes, however, these last do not clarify the real nature of the magnitudes with which the Bass-relief was elaborated.

(6) With the topographic survey developed by Sugiyama [7], he estimated that one of the sides of the Sun pyramid in Teotihuacan corresponds to: $215.72 \mathrm{~m}$. In the practice, during each of the years on the end of XIX century, the temples and all other buildings suffered refurbishment that have caused that their current lengths do not correspond with the originals. From this, the posture taken in this paper, is that the real length that is mentioned is: $216.666 \ldots \mathrm{m}$, because the justification is found in correlations that are determined with it. Nevertheless, in the plant of Sun pyramid appears in Marquina [8] on a scale of 2.65 $\mathrm{cm}=100 \mathrm{~m}$, the magnitudes in meters of the pyramid side, determined from there, it is close enough to $216.666 \ldots \mathrm{m}$. Marquina was one of the first to perform exploration and measurement of the site in Teotihuacan.

\section{REFERENCES}

[1] Jorge, Williams, Garza-Hume \& Olvera (2011). Mathematical accuracy of Aztec land surveys assessed from records in the Codex Vergara. Proceedings of the National Academy of Sciences of the United States of America.

In: http://www.ncbi.nlm.nih.gov/pmc/articles/PMC3174618/. Retrieved on May 25, 2016.

[2] Castillo, Víctor (1972). Unidades nahuas de medida. Estudios de Cultura Náhuatl, México: UNAM, Instituto de Investigaciones Históricas, 10: 195-223

[3] Harvey H. R \& Williams B. J (1980). Aztec arithmetic: positional notation and area calculation. Science, Oct 31; 210 (4469): 449-505.

[4] Dehouve, Danièle (2011). L'imaginaire des nombres chez les anciens Mexicains. Presses Universitaires de Rennes.

[5] Matos-Moctezuma, E \& López Luján, L (2010). Escultura monumental mexica. México: Fundación Conmemoraciones.

[6] Förstemann, Ernest (1880/1906). Commentar zur Mayahandschrift der koniglichen offentlichen Bibliothek zu Dresden. Trans. as Commentary on the Maya Manuscript in the Royal Public Library at Dresden. Papers of the Peabody Museum of American Archaeology and Ethnology 4/2.

[7] Sugiyama, S (2010). Teotihuacan city layout as a cosmogram. En: The archaeology of Measurement. Comprehending heaven, earth, and time in ancient societies (Edited by Iain Morley and Colin Renfrew. Cambridge University Press, pp. 130-149.

[8] Marquina, Ignacio (1951). Arquitectura prehispánica. México: Memorias del Instituto Nacional de Antropología e Historia, I.N.A.H-S.E.P, p. 71. 